\title{
Determinantes Socioeconómicos del Hambre en Países de América Latina
}

\author{
Gastón Ares (PhD) \\ Zuleika Ferre (MA) \\ Máximo Rossi (PhD)
}

Universidad de la República, Uruguay

Doi:10.19044/esj.2019.v15n16p295 URL:http://dx.doi.org/10.19044/esj.2019.v15n16p295

\section{Resumen}

El hambre puede ser definida como la sensación incómoda o dolorosa causada por el consumo insuficiente de alimentos. Es la forma más grave de inseguridad alimentaria, ya que implica que las personas no tienen acceso a suficientes alimentos para satisfacer sus necesidades básicas. A efectos de conocer con qué variables socioeconómicas está correlacionada la inseguridad alimentaria en América Latina se han estimado modelos probit ordenados utilizando los datos de la Encuesta Latinobarómetro del 2017 para 18 países latinoamericanos. En promedio, el $53 \%$ de los encuestados indicaron que nunca experimentaron falta de alimentos para satisfacer sus necesidades en los últimos 12 meses. Esto sugiere que el hambre es un problema relevante en América Latina. La heterogeneidad en la región es amplia. Mientras que el porcentaje de personas que a menudo experimentaron falta de alimentos es del 2\% para los países del sur de América Latina (Uruguay, Chile, Paraguay y Brasil), alcanza valores superiores al 10\% para varios países de Centroamérica (Honduras, República Dominicana, Panamá, Nicaragua y Venezuela). Los resultados de los modelos mostraron que la edad, el nivel educativo, la situación laboral, la raza y la privación de bienes materiales en el hogar son determinantes importantes de la prevalencia del hambre. Los programas dirigidos a mejorar el acceso a los alimentos en América Latina deben tener en cuenta estas variables para identificar a los segmentos más vulnerables de la población.

Palabras clave: Hambre, América Latina, Latinobarómetro; acceso a alimentació 


\title{
Socioeconomic Determinants of Hunger in Latin American Countries
}

\author{
Gastón Ares (PhD) \\ Zuleika Ferre (MA) \\ Máximo Rossi (PhD)
}

Universidad de la República, Uruguay

\begin{abstract}
Hunger can be defined as an uncomfortable or painful feeling caused by insufficient food intake. It is the most serious form of food insecurity, since it implies that people do not have access to enough food to satisfy their basic needs. In order to know which socioeconomic variables are correlated with food insecurity in Latin America, we have estimated probit ordered models using data from the 2017 Latinobarómetro Survey for 18 Latin American countries. On average, 53\% of respondents indicated that they have never experienced a lack of food to meet their needs in the last 12 months. This, however, suggests that hunger is a relevant problem in Latin America.

The heterogeneity in the region is wide. While the percentage of people who often experienced a lack of food is $2 \%$ for the countries of southern Latin America (Uruguay, Chile, Paraguay and Brazil), it reaches values above 10\% for several Central American countries (Honduras, Dominican Republic, Panama, Nicaragua and Venezuela).

The results of the models showed that age, educational level, work situation, race, and deprivation of material goods in the home are important determinants of the prevalence of hunger. Programs aimed at improving access to food in Latin America must take these variables into account in order to identify the most vulnerable segments of the population.
\end{abstract}

Keywords: Hunger, Latin America, Latinobarometer, access to food

\section{Introducción}

El acceso a alimentos suficientes, inocuos y nutritivos es un factor determinante de la salud y un derecho humano básico (FAO, 2013). El hambre puede definirse como la sensación incómoda o dolorosa causada por el consumo insuficiente de alimentos, el cual está relacionado con la falta de macronutrientes y micronutrientes para una vida plenamente productiva, activa y saludable (FAO, 2008; World Food Programme, 2009). Igualmente, 
el hambre es la forma más grave de inseguridad alimentaria, ya que implica que las personas no tienen acceso a alimentos suficientes para satisfacer sus necesidades básicas (FAO, 2008).

El hambre severa se ha relacionado negativamente con varios problemas de salud como enfermedades coronarias, infecciones respiratorias bajas, enfermedades diarreicas y enfermedades pulmonares crónicas (Victora et al., 2008; Bhutta et al., 2008; King et al., 2008). Además, el hambre severa plantea varios desafíos a largo plazo en el capital humano de los países al disminuir los logros escolares de las personas, la productividad del trabajo, la capacidad de aprendizaje y el bienestar general (Programa Mundial de Alimentos, 2009).

Aunque se han logrado avances sustanciales para erradicar el hambre en el mundo, aproximadamente 793 millones de personas en todo el mundo todavía luchan por cubrir su ingesta de alimentos básicos para tener una vida activa y saludable (FAO, 2015a; Butler, 2015). La gran mayoría de estas personas vive en regiones en desarrollo, como África, Asia, América Latina y el Caribe (FAO, 2015a; IFAD \& WFP, 2015a). En el caso específico de América Latina y el Caribe, si bien el desarrollo económico y las políticas exitosas de protección social han llevado a una notable reducción del hambre, el 5,5\% de la población todavía padece hambre (FAO, IFAD \& WFP, 2015a).

La erradicación del hambre para el año 2030 es una de las principales metas de los Objetivos de Desarrollo Sostenible, adoptados por las Naciones Unidas en 2015 (FAO, IFAD \& WFP, 2015b). En lo que respecta a América Latina y el Caribe, el objetivo es aún más ambicioso ya que la Comunidad de Estados Latinoamericanos y Caribeños (CELAC) adoptó en enero de 2015 el desafío de erradicar por completo el hambre para 2025 (FAO, 2015b).

Las causas del hambre son complejas, multifactoriales y varían ampliamente entre países. Estas van desde factores de corto plazo como desastres naturales, conflictos políticos, inestabilidad económica, hasta factores de largo plazo, como el crecimiento poblacional, el cambio climático y los recursos naturales limitados (Nah \& Chau, 2010). En la mayoría de los países de América Latina, los principales factores a largo plazo que subyacen al hambre no están relacionados con recursos naturales limitados (Instituto Nacional de Estadística, 2015). En este sentido, el principal desafío para erradicar el hambre en la región está relacionado con mejorar la distribución del ingreso y facilitar el acceso a alimentos suficientes, inocuos y nutritivos, particularmente de la parte más vulnerable de la población (FAO, 2015c).

Una focalización adecuada puede contribuir al desarrollo de intervenciones efectivas dirigidas a reducir el hambre y así aliviar sus consecuencias (Barret, 2010). En este sentido, la identificación de factores de riesgo, predictores y factores protectores del hambre juegan un papel clave (Pérez-Escamilla \& Segall-Corrêa, 2008). Estudios previos han identificado a 
los factores socioeconómicos como los determinantes más relevantes del acceso inadecuado a los alimentos (Corral et al., 2000; Barrett, 2010; Rossi et al., 2016). Además, se ha informado que los hogares con niños que viven en condiciones de pobreza, ubicados lejos de las zonas urbanas, encabezados por madres solteras o por minorías étnicas, corren mayor riesgo de sufrir hambre e inseguridad alimentaria. La educación ha sido identificada como un factor protector del hambre, ya que podría facilitar el acceso económico a los alimentos, mejorar las oportunidades de empleo y proporcionar conocimientos y habilidades para una mejor utilización de las fuentes de alimentos (Nah \& Chau, 2010; Bhutta, et al., 2008; Bocquier et al., 2015).

En este contexto, el objetivo del presente trabajo fue evaluar la influencia de las variables socioeconómicas sobre la prevalencia del hambre en 18 países de América Latina.

\section{Metodología}

El presente estudio utilizó la base de datos de la encuesta Latinobarómetro 2017, desarrollada por Corporación Latinobarómetro (www.latinobarometro.org). La encuesta se realizó en 18 países de América Latina: Argentina, Bolivia, Brasil, Chile, Colombia, Costa Rica, Ecuador, El Salvador, Guatemala, Honduras, México, Nicaragua, Panamá, Paraguay, Perú, República Dominicana, Uruguay y Venezuela. En cada país, se realizó un muestreo probabilístico para obtener muestras representativas nacionales de 1000-1200 encuestados. La organización proporciona un factor de ponderación para permitir la comparación de resultados entre países, independientemente del tamaño de su población. Los encuestados son mayores de 18 años en todos los países, con la excepción de Brasil y Nicaragua, donde son de más de 16 años. Se pueden encontrar detalles adicionales sobre la encuesta Latinobarómetro en http://www.latinobarometro.org/latContents.jsp.

El cuestionario incluye un total de 95 preguntas relacionadas con temas de democracia y política y 27 preguntas sociodemográficas adicionales. Una de las preguntas de este último grupo está relacionada con la prevalencia del hambre, medida como la falta de acceso a los alimentos: "En los últimos 12 meses, ¿cuán seguido usted o su familia no han tenido suficiente comida para alimentarse?" Las opciones de respuesta son: "Nunca (1), Rara vez (2), A veces (3) o Seguido (4)". Las respuestas a esta pregunta se utilizaron para estimar la influencia de las variables sociodemográficas sobre la prevalencia del hambre.

Se estimó un modelo probit ordenado para investigar la influencia de las variables socioeconómicas sobre la probabilidad de que una persona experimente falta de acceso a los alimentos. Se utilizaron las siguientes variables explicativas dummy: país, edad, sexo, estado civil, católica, 
evangelista, otras religiones, nivel de educación (analfabeto, escuela primaria incompleta, escuela primaria completa, escuela secundaria incompleta, escuela secundaria completa y universidad), ocupación y raza (blanca, afrodescendiente, mestiza e indígena). Además, se incluyó un índice de privación construido en base a la posesión de los siguientes activos: más de un dormitorio en la vivienda, propiedad de la vivienda, computadora, lavadora, teléfono residencial, celular, smartphone, automóvil, agua potable, agua caliente y aguas residuales. Los detalles de la metodología del índice de privación son proporcionados por Borooah (2001). Las estadísticas descriptivas de las variables sociodemográficas incluidas en el modelo se proporcionan en la Tabla 1.

abla 1. Estadísticas descriptivas de las variables sociodemográficas

\begin{tabular}{|c|c|c|}
\hline Descripción & Media & Desvío Estándar \\
\hline Edad & 40.607 & 16.504 \\
\hline \multicolumn{3}{|l|}{ Género } \\
\hline Mujer & 0.520 & 0.500 \\
\hline \multicolumn{3}{|l|}{ Estado civil } \\
\hline Casado & 0.533 & 0.499 \\
\hline \multicolumn{3}{|l|}{ Religión } \\
\hline Católico & 0.599 & 0.490 \\
\hline Evangelista & 0.197 & 0.397 \\
\hline Otros & 0.204 & 0.403 \\
\hline \multicolumn{3}{|l|}{ Nivel educativo } \\
\hline Sin educación formal & 0.078 & 0.268 \\
\hline Primaria incompleta & 0.172 & 0.377 \\
\hline Primaria completa & 0.172 & 0.377 \\
\hline Secundaria incompleta & 0.162 & 0.368 \\
\hline Secundaria completa & 0.213 & 0.409 \\
\hline Universidad & 0.204 & 0.403 \\
\hline \multicolumn{3}{|l|}{ Actividad laboral } \\
\hline Empleado & 0.566 & 0.496 \\
\hline \multicolumn{3}{|l|}{ Raza } \\
\hline Blanco & 0.291 & 0.454 \\
\hline Afro-descendiente & 0.065 & 0.247 \\
\hline Mestizo & 0.444 & 0.497 \\
\hline Indígena & 0.103 & 0.304 \\
\hline Índice de privación & 0.305 & 0.203 \\
\hline Observaciones totales & \multicolumn{2}{|c|}{20,010} \\
\hline
\end{tabular}

Fuente: Elaboración propia en base a la Encuesta Latinobarómetro, 2017 


\section{Resultados}

\section{La falta de acceso a los alimentos en los países de América Latina}

En promedio, el $53 \%$ de los encuestados que viven en países de América Latina nunca experimentaron falta de suficiente cantidad de comida, mientras que el 7\% indicó haber experimentado esta situación de manera seguida en los últimos 12 meses (Tabla 2). Si se suman aquellos que indican haber experimentado hambre "seguido" y "a veces", el porcentaje de personas que carecen de acceso a suficientes alimentos alcanzó al 23\% de las personas de América Latina en 2017 (Tabla 2).

Tabla 2. Proporción de respuestas a la pregunta "En los últimos 12 meses, ¿cuán seguido usted o su familia no han tenido suficiente comida para alimentarse?"

\begin{tabular}{ccccc}
\hline & Proporción & $\begin{array}{c}\text { Error } \\
\text { estándar }\end{array}$ & [95\% Intervalo de confianza] \\
\hline \hline Nunca & 0.5298 & 0.0039 & 0.5221 & 0.5375 \\
Rara vez & 0.2296 & 0.0035 & 0.2227 & 0.2365 \\
A veces & 0.1739 & 0.0028 & 0.1683 & 0.1795 \\
Seguido & 0.0668 & 0.0018 & 0.0633 & 0.0704 \\
\hline
\end{tabular}

Fuente: Elaboración propia en base a la Encuesta Latinobarómetro, 2017

Si se hace un enfoque a las dos situaciones extremas; es decir, las personas que indicaron que "seguido" no han tenido y "nunca" han tenido suficiente comida en los últimos 12 meses, se puede observar gran heterogeneidad entre los países de América Latina (Figuras 1 y 2). Venezuela fue el país que mostró el mayor porcentaje de personas que expresaron que no habían tenido suficiente comida en los últimos 12 meses $(20 \%)$, seguido de Nicaragua, Panamá, Honduras, República Dominicana y Guatemala (Figura 1). Por el contrario, Chile, Paraguay, Brasil y Uruguay tuvieron los porcentajes más bajos (entre 1 y $2 \%$ ).

En cuanto al porcentaje de personas que expresaron que en los últimos 12 meses nunca habían tenido carencia de comida; Brasil, Uruguay, Chile y Argentina mostraron los porcentajes más altos (entre $70 \%$ y $80 \%$ ). En el otro extremo, los países en peor situación con respecto al acceso suficiente a los alimentos fueron Venezuela (12\%), Nicaragua, República Dominicana y Honduras (alrededor del 35\% de la población indicó que nunca había tenido escasez de alimentos en los últimos 12 meses). En una situación ligeramente mejor se encuentra Guatemala, Perú, Panamá y México que oscilan entre 44 y $47 \%$ (sin diferencias significativas entre ellos). 
Figura 1. Porcentaje de personas que indicaron que en los últimos 12 meses de forma seguida tuvieron falta de suficiente alimento en 18 países de América Latina.

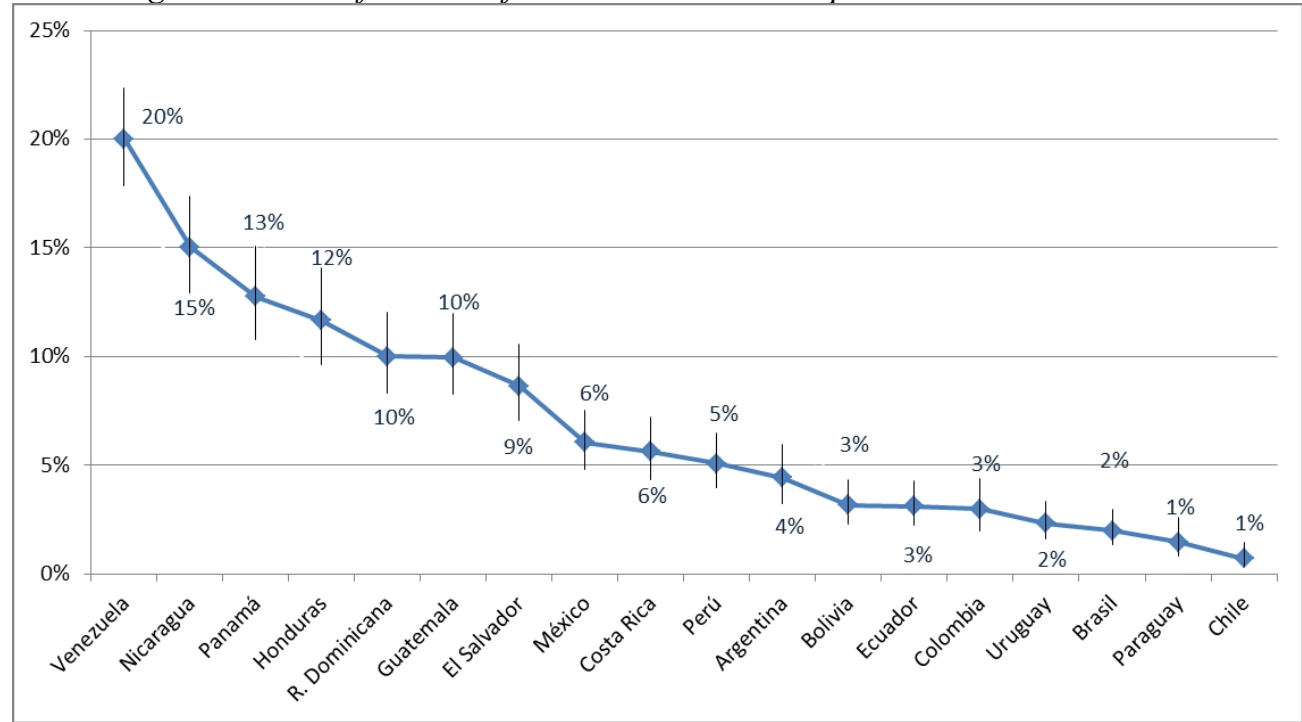

Fuente: Elaboración propia en base a la Encuesta Latinobarómetro, 2017

Figura 2. Porcentaje de personas que indicaron que nunca les ha faltado comida en los últimos 12 meses en 18 países de América Latina.

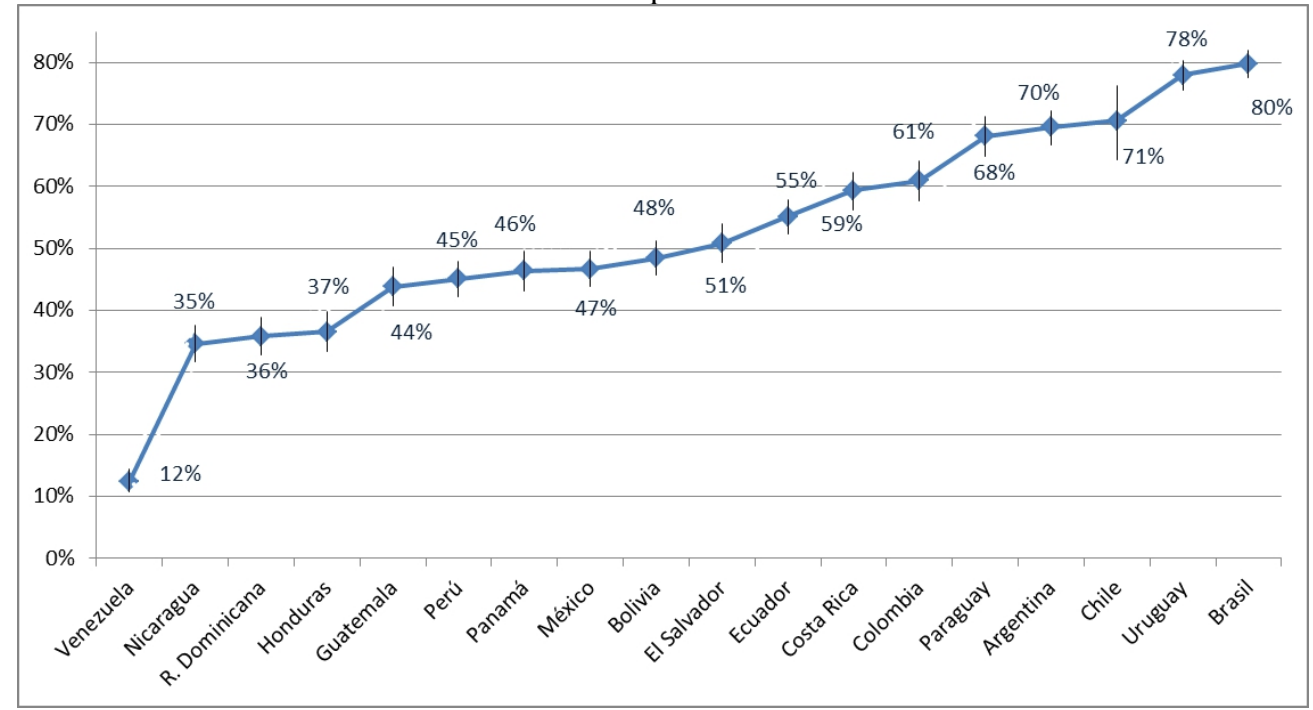

Fuente: Elaboración propia en base a la Encuesta Latinobarómetro, 2017

\section{Influencia de las variables sociodemográficas en la falta de acceso a los alimentos}

Los resultados de la estimación del modelo probit ordenado se muestran en la Tabla 3. La columna (1) presenta los coeficientes de la estimación y en las columnas (2) a (5) se presentan los efectos marginales. 
Excepto para mujeres, católicos, evangélicos y afrodescendientes, todas las variables consideradas en el modelo tuvieron un efecto significativo en la probabilidad de experimentar falta de acceso a suficiente cantidad de alimento en los últimos 12 meses. Los resultados se discuten considerando los efectos marginales con respecto a la situación "nunca haber experimentado falta de alimentos" y a la situación "haber experimentado seguido falta de alimentos".

Como se muestra en la Tabla 3, los casados, las personas mayores y las personas desfavorecidas (de acuerdo con el índice de privación) tienen más probabilidades de haber experimentado falta de suficiente cantidad de alimentos en los últimos 12 meses y menos probabilidad de nunca haber experimentado esta situación. La raza también influyó significativamente en ambas probabilidades. Ser blanco y mestizo disminuyó significativamente la probabilidad de no tener acceso a suficientes alimentos en comparación con los indígenas (Tabla 3).

De igual manera, el nivel educativo disminuyó la probabilidad de experimentar falta de acceso a suficiente cantidad de alimentos, en particular cuando los individuos completaron la escuela primaria. La probabilidad de experimentar falta de alimentos es mayor para los que no tienen educación formal. Como se muestra en la Tabla 3, el efecto protector de la educación en el hambre aumentó con el nivel educativo, como lo demuestran las diferencias en los efectos marginales.

La situación socioeconómica también tuvo una gran influencia en la probabilidad de experimentar la falta de acceso a los alimentos. Como se muestra en la Tabla 1, la probabilidad de "nunca" / "seguido" haber experimentado carencia de comida se vio significativamente influenciada por el empleo y el índice de privación.

Con respecto a la importancia relativa de las variables socioeconómicas evaluadas, la educación y el índice de privación tuvieron el mayor efecto en la probabilidad de experimentar escasez de alimentos con frecuencia. Tener un título universitario disminuyó esta probabilidad en 2.7 puntos porcentuales (pp), mientras que la privación de activos aumentó la probabilidad de inseguridad en $10.7 \mathrm{pp}$. Por el contrario, las variables con mayor efecto sobre la probabilidad de no experimentar nunca hambre fueron el índice de privación y la educación terciaria. La probabilidad de nunca experimentar falta de alimentos se redujo en 48 pp por no tener privaciones.

En cuanto a las diferencias entre países, los resultados mostraron que, con excepción de Brasil, todos los países difieren significativamente de Uruguay. Los encuestados en los 16 países restantes tenían más probabilidades de experimentar falta de acceso a suficiente comida en los últimos 12 meses que los encuestados uruguayos y menos probabilidades de nunca haber experimentado escasez de alimentos (Tabla 3). 
Tabla 3. Resultados del modelo probit ordenado que analiza la influencia de variables sociodemográficas en el acceso a los alimentos en los últimos 12 meses en 18 países de América Latina.

\begin{tabular}{|c|c|c|c|c|c|}
\hline \multirow[b]{2}{*}{ VARIABLES } & \multirow{2}{*}{$\begin{array}{c}\text { Coeficientes } \\
\text { Carencia de } \\
\text { alimentos } \\
\text { (1) }\end{array}$} & \multicolumn{4}{|c|}{ Efectos marginales } \\
\hline & & $\begin{array}{c}\text { Nunca } \\
(2) \\
\end{array}$ & $\begin{array}{c}\text { Rara vez } \\
(3) \\
\end{array}$ & $\begin{array}{c}\text { A veces } \\
(4) \\
\end{array}$ & $\begin{array}{c}\text { Seguido } \\
(5) \\
\end{array}$ \\
\hline Edad & $\begin{array}{c}0.012 * * * \\
(0.004)\end{array}$ & $\begin{array}{c}-0.005^{* * *} \\
(0.001)\end{array}$ & $\begin{array}{c}0.001 * * * \\
(0.000)\end{array}$ & $\begin{array}{c}0.002 * * * \\
(0.001)\end{array}$ & $\begin{array}{c}0.001 * * * \\
(0.000)\end{array}$ \\
\hline Edad*Edad & $\begin{array}{c}-0.000 * * * \\
(0.000)\end{array}$ & $\begin{array}{c}0.000 * * * \\
(0.000)\end{array}$ & $\begin{array}{c}-0.000 * * * \\
(0.000)\end{array}$ & $\begin{array}{c}-0.000 * * * \\
(0.000)\end{array}$ & $\begin{array}{c}-0.000 * * * \\
(0.000)\end{array}$ \\
\hline Mujer & $\begin{array}{c}0.026 \\
(0.021)\end{array}$ & $\begin{array}{l}-0.010 \\
(0.008)\end{array}$ & $\begin{array}{c}0.003 \\
(0.002)\end{array}$ & $\begin{array}{c}0.005 \\
(0.004)\end{array}$ & $\begin{array}{c}0.002 \\
(0.002)\end{array}$ \\
\hline Casado & $\begin{array}{c}-0.066 * * * \\
(0.020)\end{array}$ & $\begin{array}{c}0.026 * * * \\
(0.008)\end{array}$ & $\begin{array}{c}-0.008 * * * \\
(0.002)\end{array}$ & $\begin{array}{c}-0.013 * * * \\
(0.004)\end{array}$ & $\begin{array}{c}-0.006 * * * \\
(0.002)\end{array}$ \\
\hline Católico & $\begin{array}{l}-0.041 \\
(0.028)\end{array}$ & $\begin{array}{c}0.016 \\
(0.011)\end{array}$ & $\begin{array}{l}-0.005 \\
(0.003)\end{array}$ & $\begin{array}{l}-0.008 \\
(0.005)\end{array}$ & $\begin{array}{l}-0.004 \\
(0.003)\end{array}$ \\
\hline Evangelista & $\begin{array}{c}0.011 \\
(0.033)\end{array}$ & $\begin{array}{l}-0.004 \\
(0.013)\end{array}$ & $\begin{array}{c}0.001 \\
(0.004)\end{array}$ & $\begin{array}{c}0.002 \\
(0.006)\end{array}$ & $\begin{array}{c}0.001 \\
(0.003)\end{array}$ \\
\hline Primaria incompleta & $\begin{array}{c}-0.135 * * * \\
(0.047)\end{array}$ & $\begin{array}{c}0.053 * * * \\
(0.019)\end{array}$ & $\begin{array}{c}-0.017 * * * \\
(0.006)\end{array}$ & $\begin{array}{c}-0.025^{* * * *} \\
(0.009)\end{array}$ & $\begin{array}{c}-0.011 * * * \\
(0.004)\end{array}$ \\
\hline Primaria completa & $\begin{array}{c}-0.128 * * * \\
(0.046)\end{array}$ & $\begin{array}{c}0.051 * * * \\
(0.018)\end{array}$ & $\begin{array}{c}-0.016 * * * \\
(0.006)\end{array}$ & $\begin{array}{c}-0.024 * * * \\
(0.008)\end{array}$ & $\begin{array}{c}-0.011 * * * \\
(0.004) \\
\end{array}$ \\
\hline Secundaria incompl. & $\begin{array}{c}-0.164 * * * \\
(0.049)\end{array}$ & $\begin{array}{c}0.065 * * * \\
(0.019)\end{array}$ & $\begin{array}{c}-0.021 * * * \\
(0.007)\end{array}$ & $\begin{array}{c}-0.031 * * * \\
(0.009)\end{array}$ & $\begin{array}{c}-0.013 * * * \\
(0.004)\end{array}$ \\
\hline Secundaria completa & $\begin{array}{c}-0.261 * * * \\
(0.047)\end{array}$ & $\begin{array}{c}0.102 * * * \\
(0.018)\end{array}$ & $\begin{array}{c}-0.034 * * * \\
(0.007)\end{array}$ & $\begin{array}{c}-0.048 * * * \\
(0.008)\end{array}$ & $\begin{array}{c}-0.021 * * * \\
(0.003)\end{array}$ \\
\hline Universidad & $\begin{array}{c}-0.366^{* * *} \\
(0.049)\end{array}$ & $\begin{array}{c}0.142 * * * \\
(0.019)\end{array}$ & $\begin{array}{c}-0.049 * * * \\
(0.007)\end{array}$ & $\begin{array}{c}-0.066^{* * * *} \\
(0.008)\end{array}$ & $\begin{array}{c}-0.027 * * * \\
(0.003)\end{array}$ \\
\hline Empleado & $\begin{array}{c}-0.109 * * * \\
(0.022)\end{array}$ & $\begin{array}{c}0.043 * * * \\
(0.009)\end{array}$ & $\begin{array}{c}-0.013 * * * \\
(0.002)\end{array}$ & $\begin{array}{c}-0.021 * * * \\
(0.004)\end{array}$ & $\begin{array}{c}-0.010 * * * \\
(0.002)\end{array}$ \\
\hline Blanco & $\begin{array}{c}-0.105^{* * * *} \\
(0.031)\end{array}$ & $\begin{array}{c}0.041 * * * \\
(0.012)\end{array}$ & $\begin{array}{c}-0.013 * * * \\
(0.004)\end{array}$ & $\begin{array}{c}-0.020 * * * \\
(0.006)\end{array}$ & $\begin{array}{c}-0.009 * * * \\
(0.003)\end{array}$ \\
\hline Afro-descendiente & $\begin{array}{l}-0.051 \\
(0.045)\end{array}$ & $\begin{array}{c}0.020 \\
(0.018)\end{array}$ & $\begin{array}{l}-0.006 \\
(0.006)\end{array}$ & $\begin{array}{l}-0.010 \\
(0.008)\end{array}$ & $\begin{array}{l}-0.004 \\
(0.004)\end{array}$ \\
\hline Mestizo & $\begin{array}{c}-0.042^{*} \\
(0.026)\end{array}$ & $\begin{array}{l}0.017^{*} \\
(0.010)\end{array}$ & $\begin{array}{c}-0.005^{*} \\
(0.003)\end{array}$ & $\begin{array}{c}-0.008^{*} \\
(0.005)\end{array}$ & $\begin{array}{c}-0.004 * \\
(0.002)\end{array}$ \\
\hline Índice de privación & $\begin{array}{c}1.206^{* * * *} \\
(0.054)\end{array}$ & $\begin{array}{c}-0.479 * * * \\
(0.022)\end{array}$ & $\begin{array}{c}0.140 * * * \\
(0.007)\end{array}$ & $\begin{array}{c}0.231 * * * \\
(0.011)\end{array}$ & $\begin{array}{c}0.107 * * * \\
(0.005)\end{array}$ \\
\hline Argentina & $\begin{array}{c}0.341 * * * \\
(0.065)\end{array}$ & $\begin{array}{c}-0.135^{* * * *} \\
(0.025)\end{array}$ & $\begin{array}{c}0.028 * * * \\
(0.003)\end{array}$ & $\begin{array}{c}0.068 * * * \\
(0.013)\end{array}$ & $\begin{array}{c}0.039 * * * \\
(0.009)\end{array}$ \\
\hline Bolivia & $\begin{array}{c}0.608 * * * \\
(0.061)\end{array}$ & $\begin{array}{c}-0.235^{* * *} * \\
(0.022)\end{array}$ & $\begin{array}{c}0.032 * * * \\
(0.002)\end{array}$ & $\begin{array}{c}0.119 * * * \\
(0.011)\end{array}$ & $\begin{array}{c}0.085^{* * * *} \\
(0.012)\end{array}$ \\
\hline Brasil & $\begin{array}{c}-0.072 \\
(0.065) \\
\end{array}$ & $\begin{array}{c}0.029 \\
(0.026) \\
\end{array}$ & $\begin{array}{l}-0.009 \\
(0.008) \\
\end{array}$ & $\begin{array}{c}-0.014 \\
(0.012) \\
\end{array}$ & $\begin{array}{l}-0.006 \\
(0.005) \\
\end{array}$ \\
\hline Chile & $\begin{array}{c}0.177 * \\
(0.091)\end{array}$ & $\begin{array}{c}-0.070^{*} \\
(0.036)\end{array}$ & $\begin{array}{c}0.018 * * \\
(0.007)\end{array}$ & $\begin{array}{c}0.035^{*} \\
(0.018)\end{array}$ & $\begin{array}{l}0.018^{*} \\
(0.010)\end{array}$ \\
\hline Colombia & $\begin{array}{c}0.323 * * * \\
(0.064)\end{array}$ & $\begin{array}{c}-0.128 * * * \\
(0.025)\end{array}$ & $\begin{array}{c}0.027 * * * \\
(0.003)\end{array}$ & $\begin{array}{c}0.064 * * * \\
(0.013)\end{array}$ & $\begin{array}{c}0.037 * * * \\
(0.009)\end{array}$ \\
\hline Costa Rica & $\begin{array}{c}0.533 * * * \\
(0.062)\end{array}$ & $\begin{array}{c}-0.208 * * * \\
(0.023)\end{array}$ & $\begin{array}{c}0.032 * * * \\
(0.001)\end{array}$ & $\begin{array}{c}0.105 * * * \\
(0.012)\end{array}$ & $\begin{array}{c}0.071 * * * \\
(0.011)\end{array}$ \\
\hline
\end{tabular}




\begin{tabular}{|c|c|c|c|c|c|}
\hline R. Dominicana & $\begin{array}{c}1.010 * * * \\
(0.062)\end{array}$ & $\begin{array}{c}-0.365^{* * *} \\
(0.017)\end{array}$ & $\begin{array}{c}0.004 \\
(0.007)\end{array}$ & $\begin{array}{c}0.179 * * * \\
(0.008)\end{array}$ & $\begin{array}{c}0.182 * * * \\
(0.018)\end{array}$ \\
\hline \multirow[t]{2}{*}{ Ecuador } & $0.537 * * *$ & $-0.210 * * *$ & $0.033 * * *$ & $0.106 * * *$ & $0.071 * * *$ \\
\hline & $(0.060)$ & $(0.022)$ & $(0.001)$ & $(0.012)$ & $(0.011)$ \\
\hline \multirow[t]{2}{*}{ El Salvador } & $0.596 * * *$ & $-0.231 * * *$ & $0.030 * * *$ & $0.117 * * *$ & $0.083 * * *$ \\
\hline & $(0.067)$ & $(0.024)$ & $(0.002)$ & $(0.012)$ & $(0.013)$ \\
\hline \multirow[t]{2}{*}{ Guatemala } & $0.676 * * *$ & $-0.259 * * *$ & $0.028 * * *$ & $0.131 * * *$ & $0.100 * * *$ \\
\hline & $(0.064)$ & $(0.022)$ & $(0.003)$ & $(0.011)$ & $(0.014)$ \\
\hline \multirow[t]{2}{*}{ Honduras } & $0.775 * * *$ & $-0.293 * * *$ & $0.023 * * *$ & $0.147 * * *$ & $0.122 * * *$ \\
\hline & $(0.065)$ & $(0.021)$ & $(0.004)$ & $(0.010)$ & $(0.015)$ \\
\hline \multirow[t]{2}{*}{ México } & $0.744 * * *$ & $-0.282 * * *$ & $0.025 * * *$ & $0.143 * * *$ & $0.115^{* * *}$ \\
\hline & $(0.062)$ & $(0.021)$ & $(0.004)$ & $(0.010)$ & $(0.014)$ \\
\hline \multirow[t]{2}{*}{ Nicaragua } & $1.070 * * *$ & $-0.381 * * *$ & -0.004 & $0.184 * * *$ & $0.201 * * *$ \\
\hline & $(0.064)$ & $(0.017)$ & $(0.008)$ & $(0.007)$ & (0.019) \\
\hline \multirow[t]{2}{*}{ Panamá } & $0.828 * * *$ & $-0.310 * * *$ & $0.020 * * *$ & $0.155 * * *$ & $0.134 * * *$ \\
\hline & $(0.064)$ & $(0.021)$ & $(0.005)$ & $(0.010)$ & $(0.016)$ \\
\hline \multirow[t]{2}{*}{ Paraguay } & $0.214 * * *$ & $-0.085 * * *$ & $0.021 * * *$ & $0.042 * * *$ & $0.022 * * *$ \\
\hline & $(0.068)$ & $(0.027)$ & $(0.005)$ & $(0.014)$ & $(0.008)$ \\
\hline \multirow[t]{2}{*}{ Perú } & $0.652 * * *$ & $-0.251 * * *$ & $0.030 * * *$ & $0.127 * * *$ & $0.094 * * *$ \\
\hline & $(0.062)$ & $(0.022)$ & $(0.003)$ & $(0.011)$ & $(0.013)$ \\
\hline \multirow[t]{2}{*}{ Venezuela } & $1.659 * * *$ & $-0.509 * * *$ & $-0.081 * * *$ & $0.194 * * *$ & $0.396 * * *$ \\
\hline & $(0.056)$ & $(0.009)$ & $(0.009)$ & $(0.006)$ & $(0.021)$ \\
\hline Observaciones & 17,175 & 17,175 & 17,175 & 17,175 & 17,175 \\
\hline
\end{tabular}

Errores estándar entre paréntesis $* * * \mathrm{p}<0.01, * * \mathrm{p}<0.05, * \mathrm{p}<0.1$

\section{Discusión}

El acceso a suficientes alimentos es un derecho humano básico (FAO, 2013). Aunque se han hecho esfuerzos notables para reducir el hambre en América Latina, los resultados del presente trabajo muestran que todavía es un problema relevante. Utilizando datos auto-reportados de la encuesta Latinobarómetro 2017 en 18 países, el porcentaje de personas que a menudo no han tenido suficiente comida en los últimos 12 meses se estimó en 7\%. Este valor es cercano al de la estimación de la prevalencia del hambre proporcionada por la FAO, IFAD y WFP (2015a) para América Latina en 2014-2016 (5,5\%). Sin embargo, se debe reconocer que el compromiso político continuo ha permitido una reducción en la prevalencia del hambre de 14.7 a 5.5 en 25 años (FAO, 2015c).

Los resultados a nivel de país fueron muy similares a los datos de prevalencia del hambre proporcionados por la FAO (2015c). El hambre se considera la forma más grave de inseguridad alimentaria (FAO, 2008). En este sentido, los valores de prevalencia del hambre para varios países concuerdan con estudios previos que informaron sobre la prevalencia de inseguridad alimentaria severa (Salles-Costa et al., 2008; Muñoz-Astudillo et al., 2010; Álvarez Uribe, 2010; Bernal \& Lorenzana, 2007; Rosso et al., 2015; Pérez Escamilla et al., 2007). Sin embargo, cabe destacar que el porcentaje de 
personas que informan nunca haber experimentado carencia de alimentos no corresponde a la seguridad alimentaria, ya que no tiene en cuenta la calidad de los alimentos. En este sentido, los porcentajes reportados en la Figura 2 son superiores a la prevalencia de la seguridad alimentaria (Salles-Costa et al., 2008; Muñoz-Astudillo et al., 2010; Álvarez Uribe, 2010; Bernal \& Lorenzana, 2007; Rosso et al., 2015; Pérez Escamilla et al., 2007; Rossi et al., 2017). Un cambio dramático es el que ha ocurrido con Venezuela. En el 2013 el porcentaje de personas que indicaron que nunca les había faltado comida en los últimos 12 meses era de 58\% (elaboración propia en base a datos del Latinobarómetro 2013), mientras que en el 2017 ese porcentaje se redujo al $12 \%$.

Se encontró una gran heterogeneidad entre los países de América Latina en la prevalencia del hambre, en vista de que los porcentajes oscilan entre el 1\% y el 20\%. En particular, los países de América del Sur tienden a mostrar porcentajes más bajos de personas que dicen haber experimentado carencia de alimentos en los últimos 12 meses en comparación con los países de América Central o el Caribe. Esta marcada diferencia entre América del Sur y América Central y el Caribe se ha informado anteriormente. Según las estimaciones proporcionadas por la FAO (2015c), la región caribeña de América Latina muestra la mayor prevalencia de hambre en esa región(19,8\%), seguida de América Central (6,6\%) y finalmente América del Sur $(<5 \%)$. Estos resultados sugieren que el objetivo de erradicar completamente el hambre en la región para 2025 (FAO, 2015b) será difícil de cumplir y que las políticas que facilitan el acceso a suficientes alimentos para el segmento más vulnerable de la población siguen siendo necesarias, particularmente en varios países de América Central y el Caribe.

América Latina produce suficientes alimentos para alimentar a toda su población, lo que indica que las políticas para combatir el hambre deberían centrarse en mejorar el acceso a los alimentos y en redistribuir el poder adquisitivo de los mismos. Según Chavas (2017), esto puede lograrse mediante varias estrategias dirigidas a los segmentos más vulnerables de la población: reducir el costo de los alimentos mediante cupones de alimentos o descuentos específicos, aumentar los ingresos mediante programas de transferencia de ingresos y / o desarrollo económico, y programas de ayuda alimentaria. Estas estrategias se han implementado con éxito en Brasil y Uruguay y han llevado a reducciones marcadas en la prevalencia del hambre (da Silva et al., 2012; Ministerio de Desarrollo Social, 2015). Sin embargo, cabe destacar que no se debe centrar la atención exclusivamente en el acceso a una cantidad suficiente de alimentos, ya que la calidad nutricional también es necesaria para lograr la seguridad alimentaria (FAO, 2013).

La influencia de las variables sociodemográficas sobre la prevalencia del hambre se evaluó mediante un modelo probit ordenado. Los resultados 
mostraron que el índice de privación fue el predictor más fuerte de la prevalencia del hambre en los países de América Latina. Estudios previos también informaron sobre una fuerte asociación negativa entre la inseguridad alimentaria y el ingreso familiar en diferentes países (Bocquier et al., 2015; Carter et al., 2010; Interlenghi \& Salles-Costa, 2014; Sarlio-Lahteenkorva \& Lahelma, 2001; Rossi et al., 2017; Tingay et al., 2003). Esto es consistente con el hecho de que el hambre se ha atribuido principalmente a las desigualdades en la distribución del ingreso (Chavas, 2017) y enfatiza la importancia de implementar políticas dirigidas a mejorar la disponibilidad de dinero para la compra de alimentos en la población más vulnerable.

Igualmente, el nivel educativo influyó significativamente en la probabilidad de haber experimentado falta de alimentos en los últimos 12 meses, de acuerdo con varios estudios que informan que las personas con menos años de educación formal tienen más probabilidades de experimentar inseguridad alimentaria que las personas más educadas (Bocquier et al., 2015; Salles-Costa et al., 2008; Rossi et al., 2016; Interlenghi \& Salles-Costa, 2014; Panigassi et al., 2008). La asociación negativa entre la prevalencia del hambre y el nivel educativo puede explicarse teniendo en cuenta la relación inversa entre la educación y el estatus socioeconómico (Grusky \& Ku, 2008). En el presente trabajo, la educación muestra este efecto protector (Tabla 3).

A diferencia de otros estudios, no hemos encontrado en nuestro estudio un efecto significativo de experimentar carencia de alimentos por parte de las mujeres. Varios autores encontraron un efecto positivo y significativo (Bocquier et al., 2015; Salles-Costa et al., 2008; Carter et al., 2010; Hamelin et al., 2002; Nord et al., 2008).

Con respecto a la edad, la probabilidad de haber experimentado la falta de suficientes alimentos en los últimos 12 meses aumentó significativamente con la edad. Estudios previos realizados en países desarrollados demuestran una mayor prevalencia de personas con inseguridad alimentaria entre las personas más jóvenes o de mediana edad, lo que se ha asociado a sus ingresos más bajos (Bocquier et al., 2015; Carter et al., 2010). Sin embargo, las condiciones socioeconómicas de los adultos mayores en América Latina son diferentes a las de los países desarrollados (CEPAL, 2003). Considerando que la inseguridad alimentaria entre los adultos mayores se ha asociado a la vulnerabilidad (Pérez-Zepeda et al., 2016), los programas de alimentos dirigidos a este grupo de población parecen necesarios para facilitar el acceso a los alimentos y, en consecuencia, mejorar su estado de salud.

En el presente estudio, se encontró igualmente que la prevalencia del hambre era más alta entre los pueblos indígenas en comparación con las personas blancas y mestizas. La explicación más plausible para esta diferencia es que los pueblos indígenas generalmente tienen tasas de pobreza más altas que la población general $y$, en consecuencia, sufren más frecuentemente de 
enfermedades relacionadas con los alimentos (FAO, 2015c). Similares resultados han sido reportados por Carter et al. (2010) en lo que respecta a la inseguridad alimentaria entre las poblaciones maoríes y del Pacífico en Nueva Zelanda.

\section{Conclusión}

El objetivo del presente estudio fue analizar la prevalencia de hambre en América Latina utilizando la Encuesta Latinobarómetro 2017. En promedio, sólo el 53\% de los encuestados indicó nunca haber experimentado falta de suficiente alimento en los últimos 12 meses, lo que sugiere que el hambre sigue siendo un problema relevante en la región.

Se encontró una gran heterogeneidad entre los países. Aunque el porcentaje de personas que experimenta seguido falta de alimentos sólo se encuentra entre el 1 y el $2 \%$ para los países del sur de América Latina (Uruguay, Chile, Paraguay y Brasil), alcanzó valores superiores al 10\% para varios países de Centroamérica (Honduras, República Dominicana, Panamá, Nicaragua y Venezuela). Estos resultados evidencian la necesidad de implementar urgentemente políticas públicas dirigidas a mejorar el acceso a los alimentos en América Latina para alcanzar el objetivo de erradicar el hambre para 2025 (FAO, 2015b).

Los resultados del modelo probit ordenado mostraron que varias variables sociodemográficas influyeron en la probabilidad de experimentar carencias en el acceso a los alimentos en América Latina. En particular, la edad, el nivel educativo, la situación laboral, la raza y la privación fueron determinantes significativos de la prevalencia del hambre. Esto sugiere que los programas destinados a mejorar el acceso a los alimentos en América Latina deben tener en cuenta estas variables para identificar a los segmentos más vulnerables de la población.

\section{References:}

1. Álvarez Uribe, MC. (2010). Caracterización de los hogares de Colombia en Inseguridad Alimentaria según Calidad de Vida. Revista de Salud Pública 12, 877-888.

2. Barrett, CB. (2010). Measuring Food Insecurity. Sci 327, 825-828.

3. Bernal, J. \& Lorenzana, PA. (2007). La escala de seguridad alimentaria en hogares aplicada a adolescentes en Caracas: una medida valida y confiable. Agroalimentaria 12, 47-54., 474-481.

4. Bhutta, ZA., Ahmed, T., Black, RE., Cousens, S., Dewey, K., Giugliani, E. et al. (2008). What works? Interventions for maternal and child undernutrition and survival. The Lancet, 371(9610), 417-440.

5. Bocquier, A., Vieux, F., Lioret, S., Dubuisson, C., Caillavet, F. \& Darmon, N. (2015). Socio-economic characteristics, living conditions 
and diet quality are associated with food insecurity in France. Public Health Nutrition 18, 2952-2961.

6. Borooah, VK. (2001). Logit and Probit: Ordered and Multinomial Models. Sabe University Papers Series on Quantitative Applications in the Social Sciences. Thousand Oaks, CA: Sage.

7. Butler, C. D. (2015). Revised hunger estimates accelerate apparent progress towards the MDG hunger target. Global Food Security, 5, 1924.

8. Carter, KN., Lanumata, T., Kruse, K. \& Gorton, D. (2010). What are the determinants of food insecurity in New Zealand and does this differ for males and females? Australian and New Zealand Journal of Public Health 34, 602-608.

9. Chavas, J.P. (2017). On food security and the economic valuation of food. Food Policy, 69, 58-67.

10. Corral, L., Winters, P. \& Gordillo, G. (2000). Food Insecurity and Vulnerability in Latin America and the Caribbean. Armidale: University of New England, Graduate School of Agricultural and Resource Economics.

11. CEPAL (2003). Older persons in Latin America and the Caribbean: situation and policies. Summary. Santiago de Chile: ECLAC.

12. da Silva, J.G., Del Grossi, M.E. \& de França, C.G. (2012). Fome zero (Programa hambre cero). La experiencia brasileña. Brasilia: Ministerio de Desarrollo Agrario del Brasil.

13. FAO (2008). An introduction to basic concepts of food security. Rome: FAO; available.

14. FAO (2013). The human right to adequate food in the global strategic framework for food security and nutrition. A global consensus. Rome: FAO.

15. FAO (2015a). FAO Hunger Map 2015. Rome: FAO.

16. FAO (2015b). FAO-ECLAC: Latin America and the Caribbean is exemplary region in fight against hunger. Available at http://www.fao.org/news/story/en/item/333378/icode/ (Last accessed August 8th, 2016).

17. FAO (2015c). Regional Overview of Food Insecurity Latin America and the Caribbean. Rome: FAO.

18. FAO, IFAD \& WFP (2015a). The State of Food Insecurity in the World 2015. Meeting the 2015 international hunger targets: taking stock of uneven progress. Rome.

19. FAO, IFAD \& WFP (2015b). Achieving Zero Hunger: the critical role of investments in social protection and agriculture. Rome, FAO. 
20. Grusky, D.B. \& Ku, M.C. (2008). Gloom, Doom and Inequality. In D.B. Grusky, Social Stratification. Class, Race, and Gender in Sociological Perspective. Boulder, CO: Westview Press.

21. Hamelin, AM., Beaudry, M. \& Habicht, JP. (2002). Characterization of household food insecurity in Quebec: food and feelings. Social Science y Medicine 54, 119-132.

22. Instituto Nacional de Estadística (2015). América Latina y el Caribe sin hambre 2025. Aportes para un marco de acción. La Paz: Instituto Nacional de Estadística.

23. Interlenghi, GS. \& Salles-Costa, R. (2014). Inverse association between social support and household food insecurity in a metropolitan area of Rio de Janeiro, Brazil. Public Health Nutrition 18, 2925-2933.

24. King, D.A., Cordova, F. \& Scharf, S.M. (2008). Nutritional aspects of chronic obstructive pulmonary disease. Proceedings of the America Thoracic Society, 5, 519-523.

25. Ministerio de Desarrollo Social (2015). Pobreza, inclusión social y desigualdad en Uruguay. Avances y desafíos pendientes. Montevideo: Ministerio de Desarrollo Social.

26. Muñoz-Astudillo, MN., Martínez, JW. \& Quintero, AR. (2010). Validación de la Escala Latinoamericana y Caribeña de Seguridad Alimentaria en gestantes adolescentes. Revista de Salud Pública 12, 173-183.

27. Nah, S.L. \& Chau, C.F. (2010). Issues and challenges in defeating world hunger. Trends in Food Science y Technology, 21, 544-557.

28. Nord, M., Hooper, MD. \& Hopwood, H. (2008). Household level income-related food insecurity is less prevalent in Canada than in the United States. Journal of Hunger and Environmental Nutrition 3, 17 35.

29. Panigassi, G., Segall-Corrêa, AM., Marin-Léon, L et al. (2008). Insegurança alimentar como indicador de iniqüidade: análise de inquérito populacional. Cadernos de Saude Pública 24, 2376-2384.

30. Pérez-Escamilla, R. \& Segall-Corrêa, AM. (2008). Food insecurity measurement and indicators. Revista de Nutrição 21, 15s-26s.

31. Pérez-Escamilla, R., Melgar-Quiñonez, H., Nord, M., Álvarez Uribe, MC. \& Segall-Corrêa, AM. (2007). Escala Latinoamericana y Caribeña de Seguridad Alimentaria (ELCSA). Memorias de la 1a. Conferencia en América Latina y el Caribe sobre la medición de la seguridad alimentaria en el hogar. Perspectivas en Nutrición Humana (S),117-134; available at http://coin.fao.org/cms/media/8/13104915699830/2007_memorias_se guridad_alimentaria_medellin_pnh.pdf 
32. Pérez-Zepeda, M.U., Castrejón-Pérez, R.C., Wynne, E. \& GarciaPeña, C. (2016). Frailty and food insecurity in older adults. Public Health Nutrition, 19, 2844-2849.

33. Rossi, M., Ferre, Z., Curutchet, M.R., Giménez, A., \& Ares, G. (2017). Influence of sociodemographic characteristics on different dimensions of household food insecurity in Montevideo, Uruguay. Pub Health Nutr, 20, 620-629.

34. Rosso, M., Wicky, A., Nessier, M. \& Meyer, R. (2015). Inseguridad alimentaria en la ciudad de Santa Fe: percepción de los ciudadanos. Salud Colect. vol.11, n.2, pp. 235-245.

35. Salles-Costa, R., Pereira, RA., de Vasconcelllos MTL, da Veiga GV, de Marins, VMR., Jardim, BC., Gomes, FS. \& Sichieri, R. (2008). Association between socioeconomic factors and food insecurity: a population-based study in the Rio de Janeiro metropolitan area, Brazil. Revista de Nutrição 21, 99s-109s.

36. Sarlio-Lahteenkorva, S. \& Lahelma, E. (2001). Food insecurity is associated with past and present economic disadvantage and body mass index. $J$ Nutr 131, 2880-2884.

37. Tingay, RS., Tan, CJ., Tan, NC et al. (2003). Food insecurity and low income in an English inner city. Journal of Public Health Medicine 25, $156-159$.

38. Victora, C.G., Adair, L., Fall, C., Hallal, P. C., Martorell, R., \& Richter, L et al. (2008). Maternal and child undernutrition: consequences for adult health and human capital. The Lancet, 371(9609), 340-357.

39. World Food Programme (2009). World hunger series: Hunger and markets. Rome: World Food Programme. 